\title{
Stereoselective Alkyne Hydrogenation by using a Simple Iron Catalyst
}

\author{
Bernhard J. Gregori, ${ }^{[\mathrm{a}]}$ Felix Schwarzhuber, ${ }^{[\mathrm{b}]}$ Simon Pöllath ${ }^{[\mathrm{b}]}$ Josef Zweck, ${ }^{[\mathrm{b}]}$ Lorena Fritsch, $^{[\mathrm{c}]}$ \\ Roland Schoch, ${ }^{[c]}$ Matthias Bauer, ${ }^{*[c]}$ and Axel Jacobi von Wangelin ${ }^{*[a]}$
}

The stereoselective hydrogenation of alkynes constitutes one of the key approaches for the construction of stereodefined alkenes. The majority of conventional methods utilize noble and toxic metal catalysts. This study concerns a simple catalyst comprised of the commercial chemicals iron(II) acetylacetonate and diisobutylaluminum hydride, which enables the $Z$-selective semihydrogenation of alkynes under near ambient conditions (1-3 bar $\mathrm{H}_{2}, 30^{\circ} \mathrm{C}, 5 \mathrm{~mol} \%$ [Fe]). Neither an elaborate catalyst preparation nor addition of ligands is required. Mechanistic studies (kinetic poisoning, X-ray absorption spectroscopy, TEM) strongly indicate the operation of small iron clusters and particle catalysts.

\section{Introduction}

Precise control over the stereochemistry of alkenes is pivotal to the synthesis of numerous natural products, pharmaceuticals, fragrances, and materials. ${ }^{[1]}$ By virtue of its perfect atom economy, great operational simplicity, and versatility, the semihydrogenation of alkynes is an especially attractive tool for the stereodefined construction of alkenes. Importantly, both stereoisomers can be accessed from the same starting material by the appropriate selection of the hydrogenation catalyst. The hydrogenative synthesis of Z-alkenes has attracted significant interest owing to their special stereochemistry, higher reactivity, and a shortage of alternative methods. The partially poisoned and solid-supported Lindlar-type catalysts ( $\mathrm{Pd@CaCO}$, poisoned with quinoline and $\mathrm{Pb}(\mathrm{OAc})_{2}$ ) are the most prominent catalyst class for Z-selective semihydrogenation of alkynes. ${ }^{[2]}$ These reactions typically operate under very mild conditions, provide very high stereoselectivities, and exhibit broad functional group tolerance.

Numerous reports on the syntheses of complex natural and technical products have documented the ease of operation, scalability, versatility, and generality of this method. ${ }^{[3]}$ However, the use of the noble metal $\mathrm{Pd}$ and the presence of toxic additives (e.g., $\mathrm{PbSO}_{4}, \mathrm{PbO}$ ) constitute severe drawbacks. Alterna-

[a] B. J. Gregori, Prof. Dr. A. Jacobi von Wangelin Dept. of Chemistry, University of Hamburg Martin Luther King PI 6, 20146 Hamburg (Germany) E-mail:axel.jacobi@uni-hamburg.de

[b] F. Schwarzhuber, S. Pöllath, Prof. Dr. J. Zweck Dept. of Physics, University of Regensburg Universitätsstraße 31, 93053 Regensburg (Germany)

[c] L. Fritsch, Dr. R. Schoch, Prof. Dr. M. Bauer Institute of Inorganic Chemistry and Center for Sustainable Systems Design (CSSD), University of Paderborn

Warburger Straße 100, 33098 Paderborn (Germany) E-mail:matthias.bauer@upb.de

$\square$ Supporting Information and the ORCID identification number(s) for the

(iD) author(s) of this article can be found under: https://doi.org/10.1002/cssc.201900926. tive methods have been developed with the more abundant $3 \mathrm{~d}$ transition metals. The Nickel-P2 catalyst, prepared from nickel(II) acetate and $\mathrm{NaBH}_{4}$ in ethanol, exhibits very good stereoselectivities under mild conditions and was successfully applied to the synthesis of diverse $Z$-alkene-containing building blocks and natural products. ${ }^{[4]}$ The addition of ethylene-1,2-diamine (en) as a ligand led to improved stereocontrol. ${ }^{[4]} \mathrm{A}$ related cobalt catalyst was prepared from $\mathrm{Co}(\mathrm{OAc})_{2} \cdot 4 \mathrm{H}_{2} \mathrm{O}$, en, and $\mathrm{NaBH}_{4}$ in an $\mathrm{EtOH} / \mathrm{THF} / \mathrm{H}_{2} \mathrm{O}$ solvent mixture. ${ }^{[5]}$ In the absence of en, full hydrogenation to the alkane was observed. The development of operationally facile, inexpensive, and nontoxic procedures based on iron catalysts is highly desirable as such methods would make a significant contribution toward higher sustainability in chemical synthesis.

A few stereoselective Fe-catalyzed hydrosilylations that allow the downstream conversion of the resultant alkenylsilanes to alkenes in a separate operation have been reported $(10 \mathrm{~mol} \%$ [Fe], $10 \mathrm{~mol} \% \mathrm{PBu}_{3}, 110 \mathrm{~mol} \%$ (EtO) ${ }_{3} \mathrm{SiH}, 60^{\circ} \mathrm{C}, 48 \mathrm{~h}, \mathrm{Z} / \mathrm{E}$ up to 99:1). ${ }^{[6]}$ Stereoselective Fe-catalyzed semihydrogenations of alkynes have been largely neglected. An early protocol was reported by Taira with the use of the heterogeneous Urushibara catalyst. ${ }^{[7]}$ However, high catalyst loadings (30 mol\%) and harsh reaction conditions $\left(100^{\circ} \mathrm{C}, 50\right.$ bar $\left.\mathrm{H}_{2}\right)$ were required. High Z-stereoselectivities have been achieved in biphasic semihydrogenation of alkynes by iron nanoparticles in ionic liquids. ${ }^{[8]}$ This protocol allowed multiple catalyst recycling with minimal catalyst loss but required an elevated temperature and pressure for full conversion, most likely owing to the high viscosity, low $\mathrm{H}_{2}$ solubility, and strong NP surface coordination of the ionic liquid. ${ }^{[9]}$ To further develop the field of stereoselective Z-hydrogenation of alkynes, we believed that an effective yet operationally simple protocol would fulfill the following criteria: i) an active catalyst prepared in situ by the reduction of a simple Fe salt with a commercial reductant; ii) no complex or expensive ligands or additives; iii) highly stereoselective cata- 


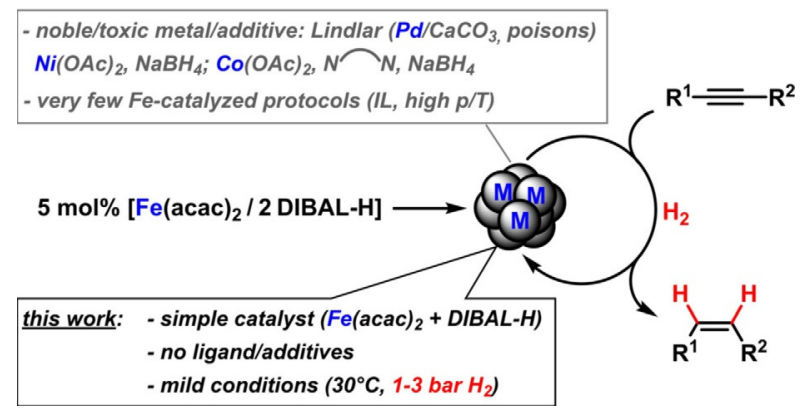

Scheme 1. Transition-metal-catalyzed Z-selective hydrogenation of alkynes.

lytic hydrogenation under mild conditions in common organic solvents (Scheme 1).

\section{Results and Discussion}

\section{Lead discovery}

Under the framework conditions, we investigated various conditions involving ferrous salts, ligands, reductants, and solvents (Table 1). Our recent investigations into the development of low-valent Fe catalysts documented the especially high activity of $\mathrm{FeX}_{2} / \mathrm{DIBAL}-\mathrm{H}$ (DIBAL-H = diisobutylaluminum hydride) catalyst mixtures in alkene hydrogenations. ${ }^{[10]}$ Such catalysts also proved to be effective in the hydrogenation of the model substrate phenylprop-1-yne. $\mathrm{FeCl}_{2}$ (THF) and $\mathrm{Fe}(\mathrm{hmds})_{2}$ (hmds= hexamethyldisilazide, $\left.\mathrm{N}\left(\mathrm{SiMe}_{3}\right)_{2}\right)$ formed very effective complete hydrogenation catalysts that exclusively gave the alkane (entries 1-3). At a lower DIBAL-H concentration, Fe(hmds) 2 was

\begin{tabular}{|c|c|c|c|c|}
\hline $\mathrm{Ph}-$ & $=\mathrm{Me} \stackrel{x}{=}$ & $\begin{array}{r}5 \mathrm{~mol} \% \mathrm{~N} \\
x \mathrm{~mol} \% \text { DIB } \\
2{\mathrm{bar} \mathrm{H}_{2,}}^{7} \\
30^{\circ} \mathrm{C}, 3\end{array}$ & $\underset{\mathrm{h}}{\stackrel{\mathrm{AL}-\mathrm{H}}{\longrightarrow}} \underset{\mathrm{Ph}}{\stackrel{\mathrm{CX}}{\longrightarrow}} \underset{\mathrm{Me}}{+}$ & $=_{(E)}^{\mathrm{Me}}+\overbrace{\mathrm{Ph}}^{\mathrm{Me}}$ \\
\hline Entry & $\mathrm{MX}_{2}$ & $x[\mathrm{~mol} \%]$ & Alkene yield $[\%]^{[\mathrm{a}]} Z / E^{[\mathrm{a}]}$ & Alkane yield $[\%]^{[a]}$ \\
\hline 1 & $\mathrm{FeCl}_{2}(\mathrm{thf})_{1.5}$ & 510 & $0^{[\mathrm{b}]}$ & $>99$ \\
\hline $2^{[\mathrm{b}]}$ & $\mathrm{Fe}(\mathrm{hmds})_{2}$ & 5 & $<1$ & 7 \\
\hline 3 & & 10 & $<1$ & $>99$ \\
\hline 4 & $\mathrm{Fe}(\mathrm{OAC})_{2}$ & 5 & $14: 1$ & 6 \\
\hline 5 & & 10 & $1: 20$ & 76 \\
\hline 6 & $\mathrm{Fe}(\mathrm{acac})_{2}$ & 5 & $14: 1$ & $<1$ \\
\hline 7 & & 10 & $18: 1$ & $<1$ \\
\hline $8^{[c]}$ & & 10 & $5: 1$ & $<1$ \\
\hline 9 & & 15 & $<1$ & $>99$ \\
\hline 10 & & 20 & $<1$ & $>99$ \\
\hline 11 & $\mathrm{Ni}(\mathrm{acac})_{2}$ & 10 & $<1$ & $>99$ \\
\hline 12 & $\mathrm{Co}(\mathrm{acac})_{2}$ & 10 & $<1$ & $>99$ \\
\hline $13^{[d]}$ & $\mathrm{Cu}(\mathrm{acac})_{2}$ & 10 & $<1$ & 0 \\
\hline \multicolumn{5}{|c|}{$\begin{array}{l}\text { Conditions: } 0.2 \mathrm{mmol} \text { alkyne, } 0.4 \mathrm{~mL} \text { THF. [a] Determined by }{ }^{1} \mathrm{H} \text { NMR and } \\
\text { GC-FID. [b] Cyclotrimerization as major reaction. [c] Sequential addition of } \\
\mathrm{Fe}(\mathrm{acac})_{2} \text { and DIBAL-H to alkyne/THF solution. [d] } 5 \mathrm{~h} \text {. }\end{array}$} \\
\hline
\end{tabular}

an active catalyst of the $[2+2+2]$-cyclotrimerization to the benzene derivatives. $^{[11]}$ At higher DIBAL-H concentrations, the Fe catalysts rapidly formed black suspensions, which efficiently catalyzed the complete hydrogenation to the alkane (entries 1, $3,5,9,10)$. The combination of commercial $\mathrm{Fe}(\mathrm{acac})_{2}$ with DIBAL-H (molar ratio 1:2) in THF exhibited the highest catalytic activity at only 2 bar $\mathrm{H}_{2}$ pressure and $30^{\circ} \mathrm{C}$ toward semihydrogenation ( $98 \%$ yield of alkenes) with very high stereoselectivity $(Z / E=18: 1$, Table 1). Importantly, the $Z / E$ ratios remained constant over the course of the reaction and only slightly decreased at long reaction times owing to competing $Z-E$ isomerization and preferred $Z$-alkene hydrogenation (over $E$ alkene).

\section{Substrate scope}

The optimized reaction conditions were then successfully applied to the semihydrogenation of diaryl alkynes even at a near-ambient pressure of only 1.3 bar $\mathrm{H}_{2}$ (Table 2). The general

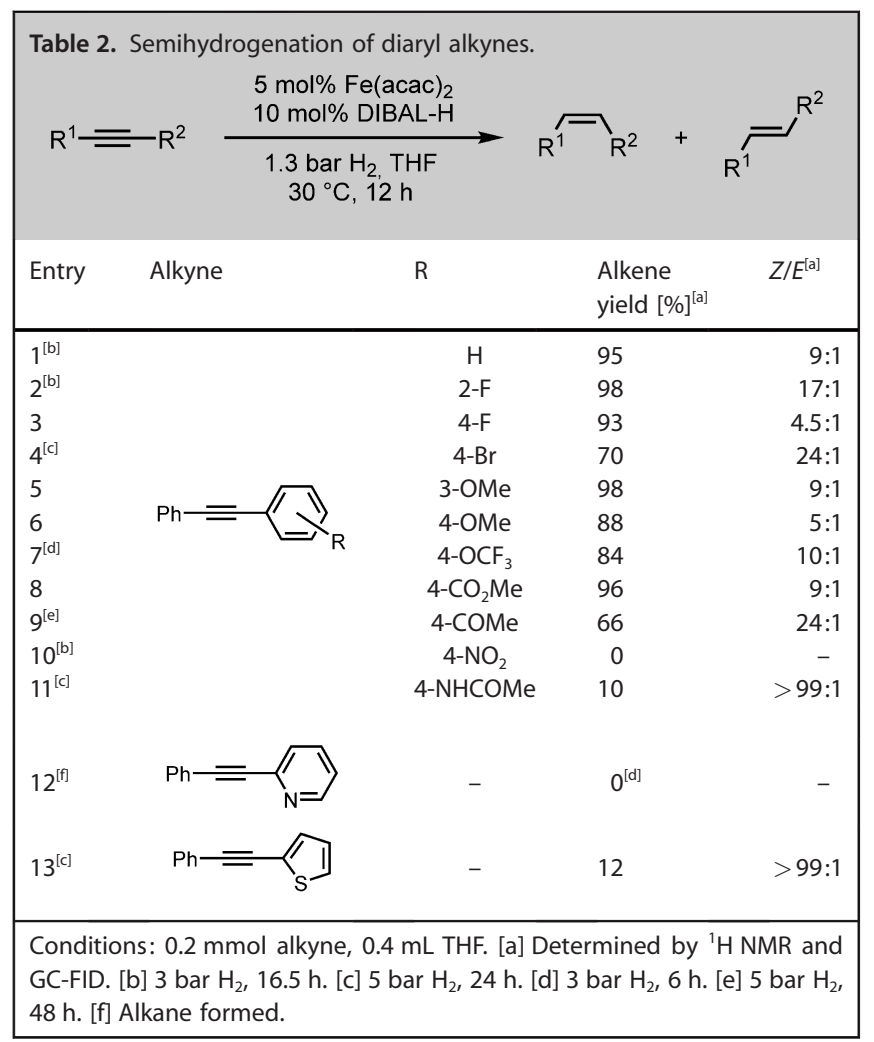

protocol tolerated various substitution patterns and exhibited good functional group compatibility, for example, carbonyls, halides, esters, and amines. Highly electrophilic substituents such as nitro, cyano, pyridyl, and acidic protons were not tolerated. ${ }^{[1,5,12]}$ Full conversions were mostly observed (entries 17); (4-bromo-phenyl) ethynylbenzene gave high Z-selectivity and minor formation of dehalogenation products (entry 4). ${ }^{[13]}$ No hydro-dehalogenation was observed with fluoro and chloro derivatives. No competing reduction of carbonyl/carboxyl groups occurred (entries 8 and 9). The nitrophenyl derivative 
underwent nitro reduction (equimolar to catalyst concentration) and led to decomposition of the catalyst (entry 10). (2Pyridyl)ethynyl-benzene exclusively afforded the alkane product; the thiophene substrate exhibited very high stereoselectivity but low conversion (entries 12, 13). ${ }^{[5]}$ Alkyl-substituted alkynes were also competent substrates in the Z-selective semihydrogenation (Table 3). Terminal alkynes underwent rapid

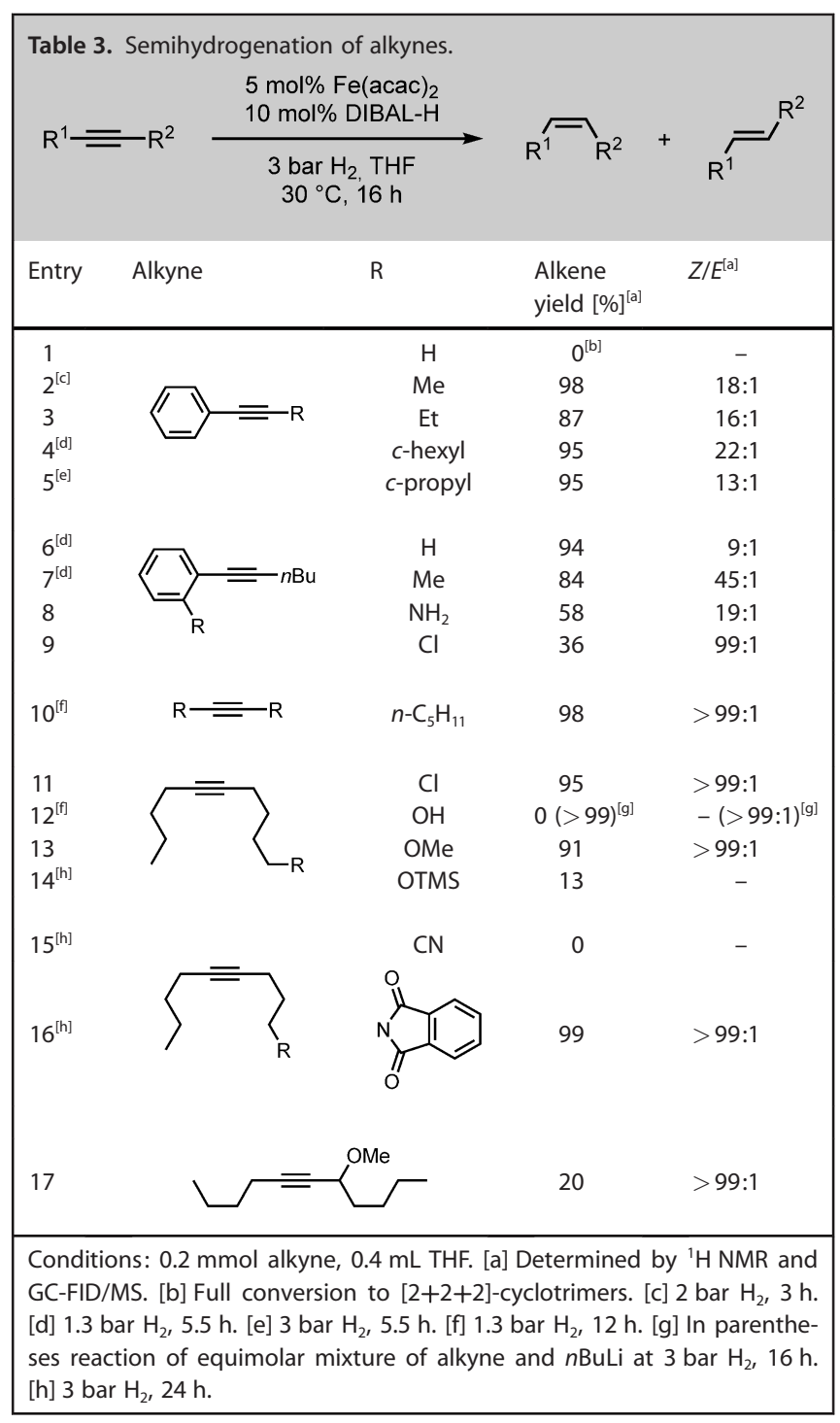

$[2+2+2]$-cyclotrimerization to the benzene derivatives rather than hydrogenation (entry 1,99\%). The $\mathrm{NH}_{2}$ group of 1-(2-aminophenyl)-1-hexyne was fully tolerated (entry 8). Generally, reactions with 1-alkyl alkynes displayed significantly higher stereocontrol than 1-aryl alkynes. The highest yields and stereoselectivities were achieved with internal alkyl-substituted alkynes. The 1-trimethylsilyl group gave moderate yields and Z/E ratios (Scheme 2, top) whereas the bulky triisopropyl-silyl (TIPS) substituent inhibited conversion. The general conditions were applied to the synthesis of two natural products. Semihydrogenation of 5-decynyl-1-acetate and 9-tricosyne resulted in complete conversions to the $Z$-alkenes in very high yields and per-

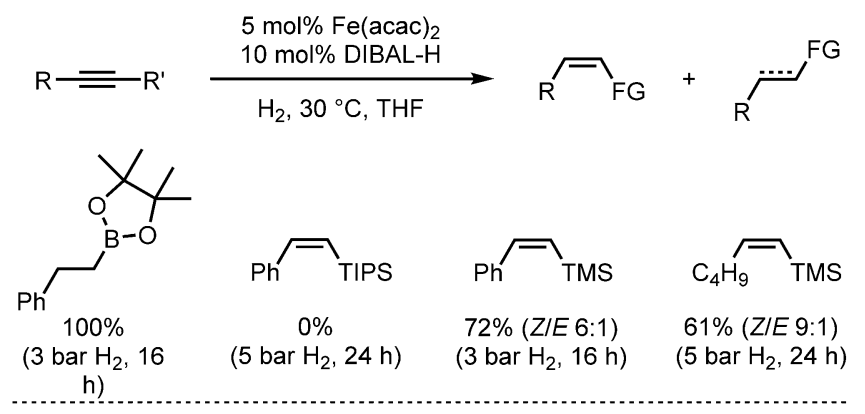

insect pheromone of the turnip moth Agrotis segetum
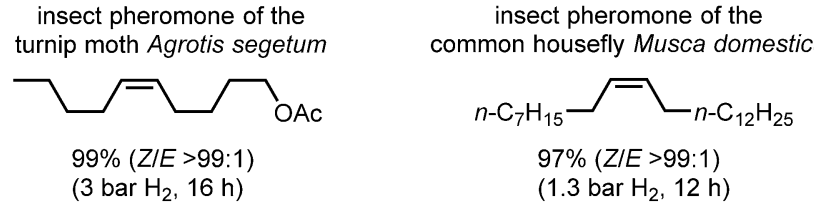

Scheme 2. Top: Semihydrogenation of functionalized alkynes. Bottom: Synthesis of two insect pheromones. Yields and isomer ratios were determined by ${ }^{1} \mathrm{H}$ NMR spectroscopy and GC-FID.

fect stereoselectivities (Scheme 2, bottom). The 1-acetate product is the natural insect pheromone of the turnip moth Agrotis segetum; ${ }^{[4 d]}$ 9-Z-tricosene is the insect pheromone of the common housefly Musca domestica. ${ }^{[14]}$

\section{Kinetic studies}

The reaction order in catalyst [Fe] was determined to be approximately 1.4 at low catalyst concentrations $(5-25 \mathrm{~mm})$ and decreased to approximately 0.7 at higher concentrations (25$50 \mathrm{~mm}$ ). The reducing conditions in the presence of the co-catalyst DIBAL-H and the stoichiometric reductant dihydrogen $\mathrm{H}_{2}$ suggest the involvement of low-valent catalyst species, which are very likely to aggregate to larger particles in the absence of stabilizing ligands. The unambiguous distinction between homotopic and heterotopic catalysis mechanisms is very intricate. ${ }^{[15]}$ We performed kinetic poisoning experiments, which were consistent with a heterogeneous mechanism. ${ }^{[15 \mathrm{a}]} \mathrm{Hg}$ poisoning (addition of 200 equiv. $\mathrm{Hg}$ per [Fe] $2 \mathrm{~h}$ prior to start of hydrogenation reaction) showed a slight loss of catalyst activity (Figure 1). ${ }^{[15 d]}$ The heterogeneity of the catalyst was further indicated by experiments in the presence of trimethyl-phosphite $\left(\mathrm{P}(\mathrm{OMe})_{3}\right) \cdot{ }^{[15]}$ Complete catalyst inhibition of a standard reaction with $5 \mathrm{~mol} \%$ [Fe] loading was achieved with only $1 \mathrm{~mol} \% \mathrm{P}(\mathrm{OMe})_{3}$ (addition after 30 min reaction), which corresponds to a particle size of approximately $10 \mathrm{~nm}$. At higher $\mathrm{P}(\mathrm{OMe})_{3}$ concentrations, the reestablishment of moderate catalyst activity was observed, most likely as a consequence of the leaching of catalyst species into the bulk solvent phase. A similar effect was recorded when dibenzo[a,e]cyclooctatetraene (dct), a selective poison of low-valent homogeneous metal species, was used. ${ }^{[15 b, c]}$ Upon the addition of $30 \mathrm{~mol} \%$ dct to the reaction at $30 \mathrm{~min}$, the catalyst was deactivated to approximately $2 / 3$ of its original efficiency. From these observations, we deduced that heterotopic or nanoparticulate catalyst spe- 


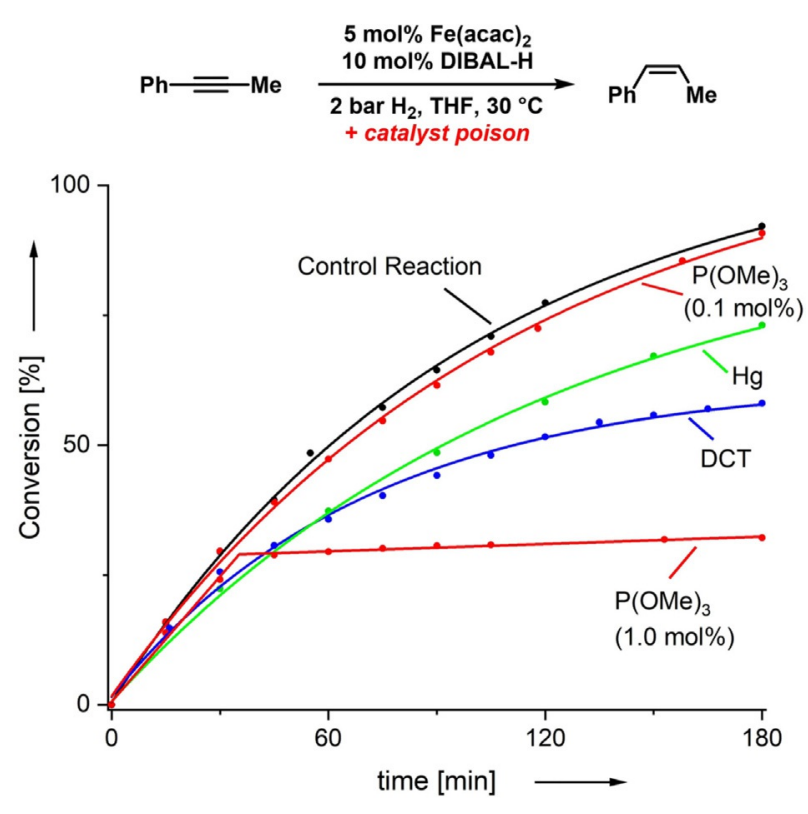

Figure 1. Catalyst poisoning experiments with 200 equiv. $\mathrm{Hg}$ per [Fe] (addition during catalyst formation and $2 \mathrm{~h}$ prior to hydrogenation), 6 equiv. DCT per [Fe] (addition after 30 min of hydrogenation), and 0.02-0.2 equiv. $\mathrm{P}(\mathrm{OMe})_{3}$ per $[\mathrm{Fe}]$ (addition after $30 \mathrm{~min}$ of hydrogenation), respectively.

cies were operative, possibly in equilibrium with homotopic soluble catalyst species.

\section{Catalyst characterization}

As an element-specific and short-range sensitive method, $X$-ray absorption spectroscopy is a powerful tool to obtain information about the oxidation state and coordination environment of the absorbing atoms in solution and thus highly instructive toward the investigation of catalyst species. ${ }^{[16]}$ We performed $X$-ray absorption near-edge structure (XANES) and extended Xray absorption fine structure (EXAFS) analysis of the catalyst combinations: $\mathrm{Fe}(\mathrm{hmds})_{2} / \mathrm{DIBAL}-\mathrm{H}$ and $\mathrm{Fe}(\mathrm{acac})_{2} / \mathrm{DIBAL}-\mathrm{H}^{\left[{ }^{[17]}\right.}$ The former catalyst contained a bulky, redox-stable amido ligand that was easily displaced by a formal hydride delivered from DIBAL-H. The resultant catalyst exhibited very high activity in the complete hydrogenation of alkynes to the alkanes and the $[2+2+2]$-cyclotrimerization. ${ }^{[10,11]}$ The latter catalyst formed from $\mathrm{Fe}(\mathrm{acac})_{2}$ and DIBAL-H enabled highly selective semihydrogenation to Z-alkenes. However, the presence of reduction-sensitive carbonyl moieties within the acetylacetonate ligand can alter the mode of action of the co-catalyst DIBAL-H (vide infra). The XANES spectra unambiguously show the reduction of both $\mathrm{Fe}(\mathrm{acac})_{2}$ and $\mathrm{Fe}(\mathrm{hmds})_{2}$ upon addition of DIBAL-H (Figure 2). ${ }^{[17]}$ Although comparably small changes of the edge energies of maximum of $1.7 \mathrm{eV}$ were observed for $\mathrm{Fe}(\mathrm{acac})_{2}$, the reduction of the whiteline intensity accompanied by an increased prepeak intensity and broadening at $7113 \mathrm{eV}$ indicated an oxidation state of $\mathrm{Fe}^{\mathrm{l}}$ (or lower) in the final reduction state (after addition of 4 equivalents (equiv.) DIBAL-H) and the formation of agglomerates, in agreement with previous studies. ${ }^{[16]}$ For $\mathrm{Fe}(\mathrm{acac})_{2}$, the pronounced whiteline was only

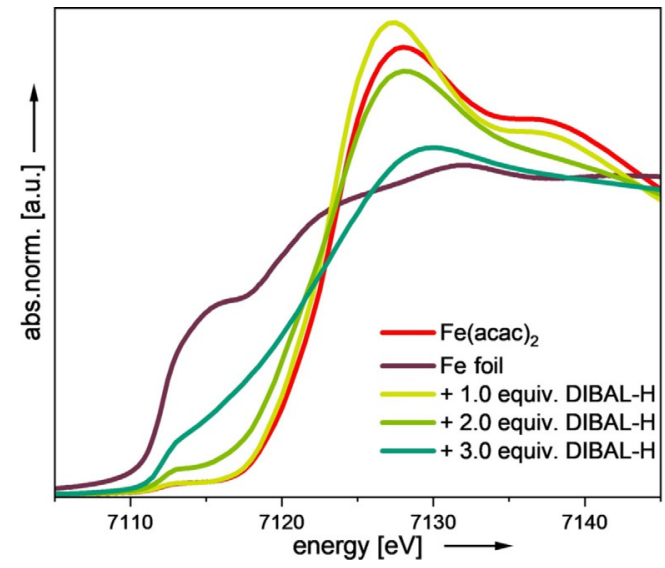

Figure 2. XANES spectra of Fe(acac) ${ }_{2}$ and after the addition of DIBAL-H.

significantly reduced after addition of 3 equivalents of DIBAL$\mathrm{H}$, which corresponded to a higher concentration of the reductant than the catalytically active species formed from $\mathrm{Fe}(\mathrm{acac})_{2}$ and 2 equivalents of DIBAL-H. In contrast, the Fe(hmds) ${ }_{2}$ spectrum, which was significantly different to unreduced $\mathrm{Fe}(\mathrm{acac})_{2}$ possibly owing to the presence of pronounced $\pi$-resonance in the $\mathrm{Fe}-\mathrm{N}$ bonds, changed with 0.5 equivalents of DIBAL-H. ${ }^{[17]}$ Interestingly, the XANES spectra of $\left[\mathrm{Fe}(\mathrm{hmds})_{2}+2\right.$ equiv. DIBAL$\mathrm{H}]$ and $\left[\mathrm{Fe}(\mathrm{acac})_{2}+4\right.$ equiv. DIBAL-H] showed very similar spectral shapes. ${ }^{[17]}$ This is in full accord with their identical catalytic performance as (complete) hydrogenation catalysts that exclusively deliver the alkane product (see Table 1, entries 3 and 9). Therefore, both catalyst species were closely related in their structural and electronic properties and two acac ligands "quenched" 2 equivalents of DIBAL-H to give alcoholates.

The EXAFS data showed that both pre-catalysts $\mathrm{Fe}(\mathrm{hmds})_{2}$ and $\mathrm{Fe}(\mathrm{acac})_{2}$ formed dimers upon addition of DIBAL-H and even larger aggregates with increasing equivalents of the reductant. The maximum Fe-Fe coordination number was 3 at a Fe-Fe distance of $2.5 \AA$, corresponding to at least tetranuclear agglomerates, ${ }^{[17]}$ which formed from $\mathrm{Fe}(\mathrm{acac})_{2}$ with 3 equivalents of DIBAL-H and from $\mathrm{Fe}(\mathrm{hmds})_{2}$ with 1 equivalent of DIBAL-H. A comparison of the EXAFS data in Figures 3 and 4

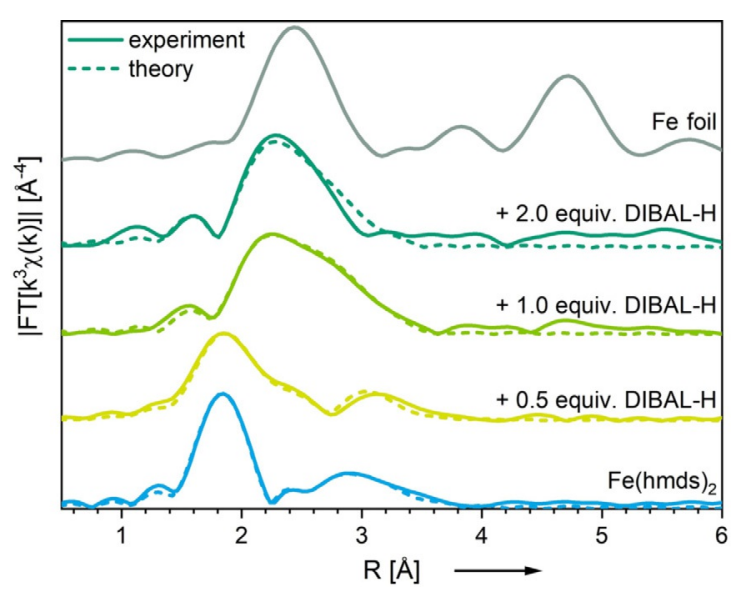

Figure 3. EXAFS spectra in R-space of $\mathrm{Fe}(\mathrm{hmds})_{2}$ with addition of DIBAL-H. 


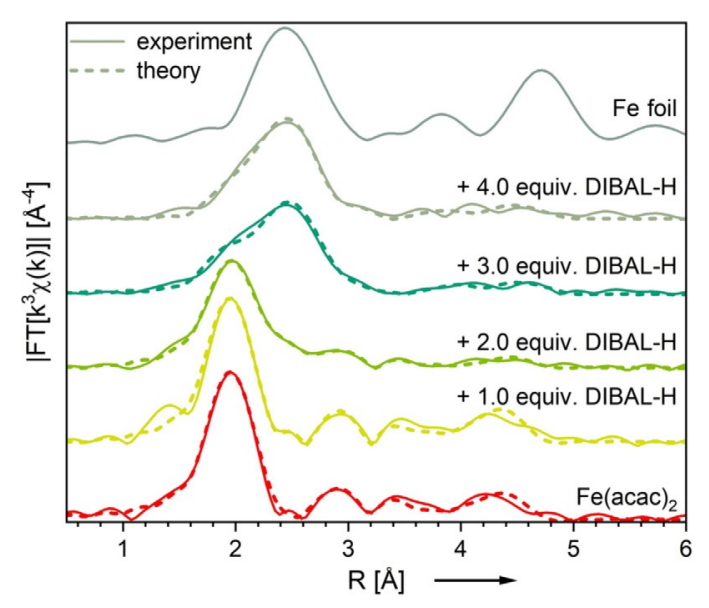

Figure 4. EXAFS spectra in R-space of $\mathrm{Fe}(\mathrm{acac})_{2}$ with addition of DIBAL-H.

documents the much less effective Fe-centered reduction of $\mathrm{Fe}(\mathrm{acac})_{2}$ by DIBAL-H, in agreement with the XANES analyses. Further results of our EXAFS study are: The absorption fine structure of the highly selective semihydrogenation catalyst $\left[\mathrm{Fe}(\mathrm{acac})_{2}+2\right.$ equiv. DIBAL-H] was significantly different from the bulk $\mathrm{Fe}^{0}$ foil reference. The presence of the Fe-O contribution resulting from the $[\mathrm{acac}]^{-}$(or derivatives thereof) was only observed with up to 2 equivalents of DIBAL-H. With higher DIBAL-H concentration, an abrupt change to shorter Fe-O distances and lower Fe-O coordination numbers (from 4-6 to 12) was observed, which was in agreement with the XANES results. We believe that coordination of a chemically different $\mathrm{O}$ donor ligand was responsible for the new Fe-O contribution (e.g., the solvent THF or alcoholate derivatives formed from hydride addition to acac). In both catalyst series, the addition of
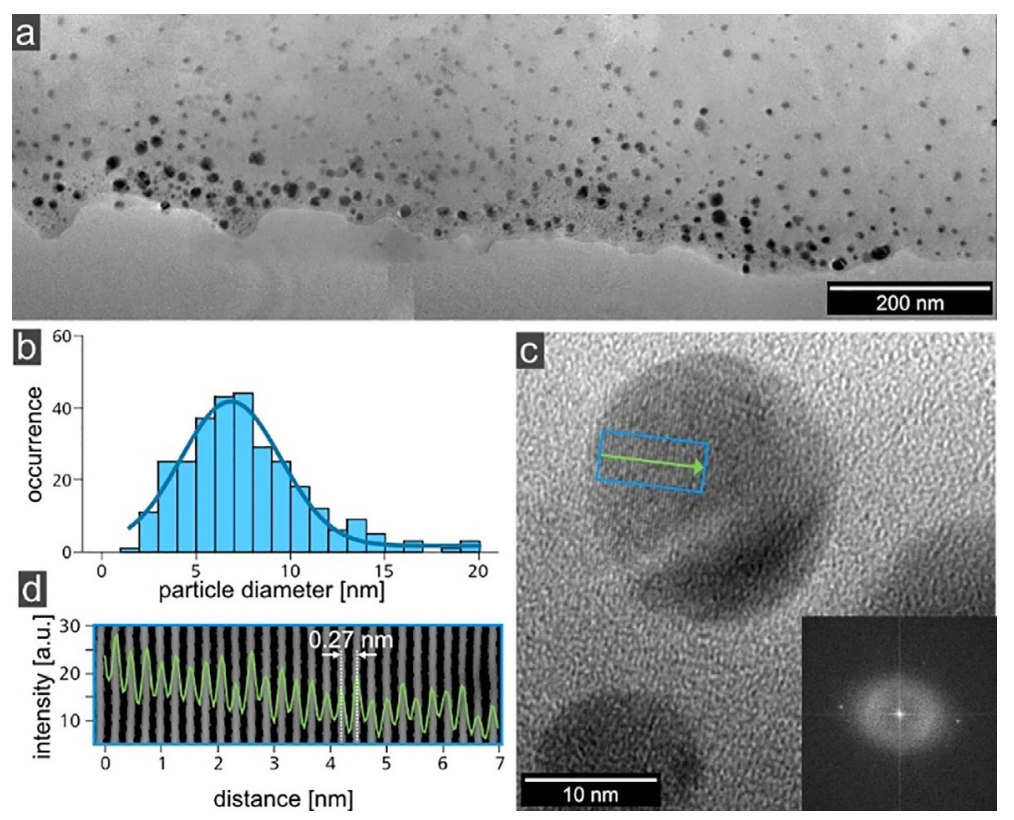

Figure 5. TEM measurements. (a) Nanoparticles embedded in residual matter from solvent, acac, DIBAL-H. (b) Size distribution of 300 particles. (c) High-resolution TEM of separated particles showing atom fringes; inset: corresponding diffractogram. (d) Line scan along the atom fringes shown in c (along green arrow). further DIBAL-H did not significantly alter the nature of the catalyst species. The requirement of higher DIBAL-H stoichiometry for the generation of an equally active (complete) hydrogenation catalyst from Fe(acac) $)_{2}>3$ equiv. DIBAL-H) supported the notion that there was concomitant ligand reduction operative. ${ }^{[17]}$

We used TEM to prove the nature, size, and shape of the potentially heterogeneous catalysts. In accordance with the kinetic poisoning experiments, the TEM spectra documented the presence of $\mathrm{Fe}$ particles from the reduction of $\mathrm{Fe}(\mathrm{acac})_{2}$ with 2 equivalents of DIBAL-H and after vacuum treatment (Figure 5). A TEM image with a $0.3 \times 1.2 \mu \mathrm{m}$ field of view containing many separate particles embedded in organic remnants is displayed in Figure $5 \mathrm{a}$. The distribution of the particle size gave an average particle diameter of $6.9 \mathrm{~nm}$ with a standard deviation of $2.6 \mathrm{~nm}$ (Figure $5 \mathrm{~b}$ ). ${ }^{[17]}$ A high-resolution TEM image proved the particles' crystallinity (Figure $5 c$, see diffractogram in inset). A line scan along the green arrow with an integration width of $3.6 \mathrm{~nm}$ is displayed in Figure $5 \mathrm{~d}$. The lattice constant, averaged over 10 fringes, was $0.27 \pm 0.02 \mathrm{~nm}$, which was in full agreement with the presence of elemental $\mathrm{Fe}^{0} .{ }^{[18]}$

\section{Reaction mechanism}

We collected several synthetic, kinetic, and spectroscopic data to characterize the operating catalysts species under reaction conditions. The optimal stoichiometries of the $\mathrm{FeX}_{2}$ pre-catalysts and the reductant DIBAL-H that form an active and selective semihydrogenation catalyst appear to exclude $\mathrm{Fe}^{0}$ particle catalysts (Table 1). With 2 equivalents of DIBAL-H (which could affect $\mathrm{FeX}_{2} \rightarrow \mathrm{Fe}^{0}$ reduction), the ferrous precatalysts $\mathrm{Fe}(\mathrm{hmds})_{2}$, $\mathrm{FeCl}_{2}, \mathrm{Fe}(\mathrm{OAC})_{2}$ catalyze the complete hydrogenation to the alkane instead. Contrary to these observations, an active semihydrogenation catalyst was formed from $\mathrm{Fe}(\mathrm{acac})_{2} / \mathrm{DIBAL}-\mathrm{H}$ in a $1 / 2$ ratio, which was a direct consequence of the hydride buffering effect of acac. Consistent with this hypothesis is the detection of reduced side products derived from hydride addition to acac. The addition of acetophenone exerted a similar buffering effect (Scheme $3 \mathrm{~A}$ ). Related reduction products from the reaction of tri(benzoylacetonato) iron(III) $\left(\mathrm{Fe}(\mathrm{bzac})_{3}\right)$ and DIBAL-H were isolated in approximately $100 \%$ yield (Scheme $3 \mathrm{~B}$ ). XANES and EXAFS spectra were in full support of the initial hydride transfer to the ligand rather than the Fe centers. However, we cannot exclude the secondary transfer of a formal hydride anion through $\beta$-hydride elimination of acac-derived alcoholates at Fe. XANES and EXAFS data further suggested that the catalytic species were most likely not as far reduced as an $\mathrm{Fe}^{0}$ species but involved smaller metal nanoclusters bearing $\mathrm{Fe}-\mathrm{O}$ coordination motifs (acac or reduction products thereof). The kinetic poisoning studies suggested an equilibrium between homogeneous and heterogeneous catalysts. A similar result can be deduced from the reaction order in the catalyst, which ranged from approximately $1.4(1-5 \mathrm{~mol} \% \mathrm{Fe})$ to 0.7 


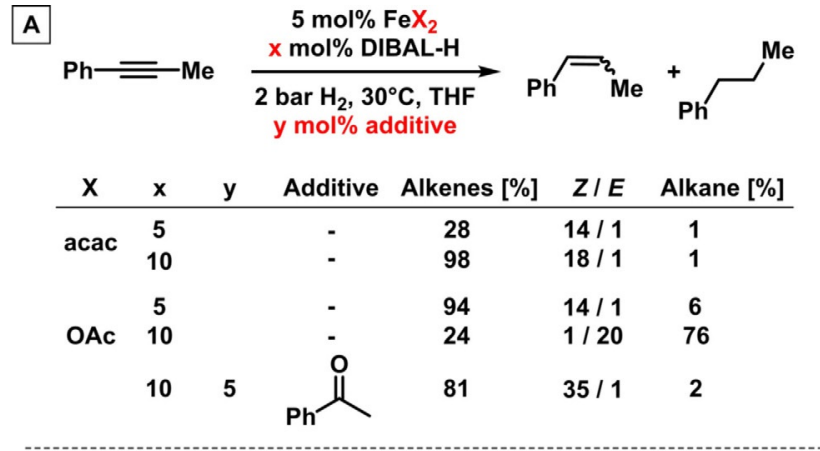

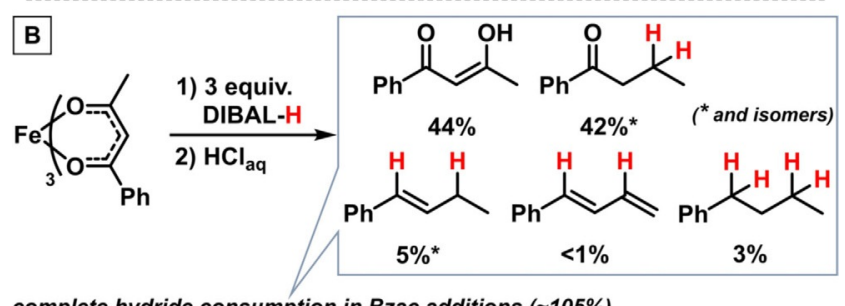

complete hydride consumption in Bzac additions ( 105\%)

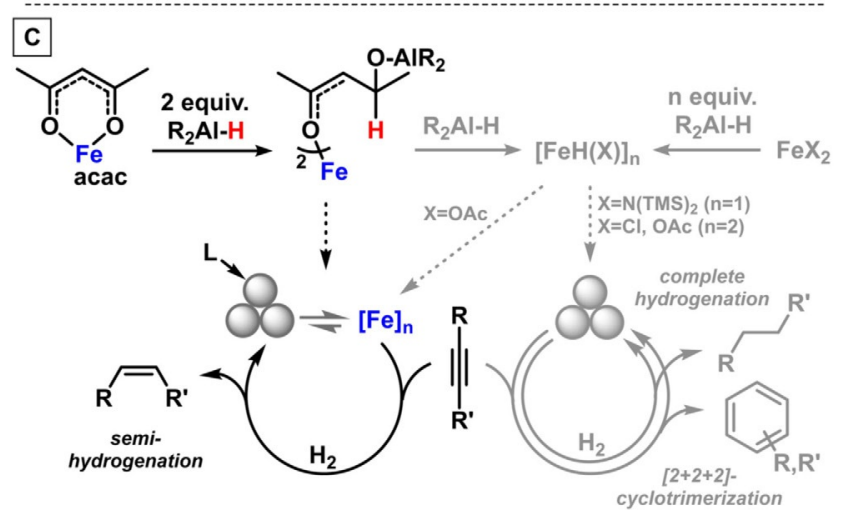

Scheme 3. (A) Carbonyl additive effect; (B) Hydride addition products of $\mathrm{Fe}(\mathrm{bzac})_{3}$; (C) Postulated mechanism involving acac-reduction by DIBAL-H.

$(5-10 \mathrm{~mol} \% \mathrm{Fe}) .{ }^{[17]}$ Fe particles were detected under the ex situ conditions of the TEM measurements. The combined data from visual inspections of the hydrogenation reactions, kinetic studies, poisoning experiments, XANES, EXAFS, and TEM measurements led to a mechanistic proposal that involves initial hydride addition to the acac-ligands in $\mathrm{Fe}(\mathrm{acac})_{2}$ and subsequent formation of a Fe cluster and particle catalysts (Scheme $3 \mathrm{C}$ ).

\section{Conclusions}

An operationally facile and highly efficient iron-catalyzed semihydrogenation of alkynes was developed. The simple, inexpensive catalyst combination $\mathrm{Fe}(\mathrm{acac})_{2} / \mathrm{DIBAL}-\mathrm{H}$ enabled the synthesis of various $Z$-alkenes in high yields and with high stereocontrol under very mild conditions ( $1-3$ bar $\mathrm{H}_{2}, 30^{\circ} \mathrm{C}$ ). Mechanistic studies were indicative of smaller metal nanoclusters and nanoparticles as active catalyst species. For the first time, X-ray absorption spectroscopy was used to study the reductive formation of the catalyst. The superior activity and selectivity of catalyst species formed through ligand-centered hydride reduction of acac rather than the direct hydride coordination to the metal center is a hitherto unknown effect in such hydrogenation reactions and worthy of further investigations. The use of commercial catalysts and reagents and the absence of any expensive, toxic, or complex ligand makes this catalytic semihydrogenation attractive for lab-scale and larger scale operations.

\section{Experimental Section}

\section{Catalyst preparation}

Under an atmosphere of argon (Schlenk technique or glove box), an oven-dried vial was charged with a stir bar and $\mathrm{Fe}(\mathrm{acac})_{2}$ $(6.4 \mathrm{mg}, 25 \mu \mathrm{mol})$, and dry THF $(0.95 \mathrm{~mL})$ was added. The mixture was stirred for $10 \mathrm{~min}$ at RT and DIBAL-H was added dropwise ( $50 \mu \mathrm{L}, 1.0 \mathrm{M}$ in toluene, $50 \mu \mathrm{mol}, 2.0$ equiv.). The resultant black mixture was stirred for $20 \mathrm{~min}$ before use.

\section{Catalytic semihydrogenation}

Under an atmosphere of argon (Schlenk technique or glove box), an oven-dried $4 \mathrm{~mL}$ vial with a stir bar was charged with the alkyne $(0.2 \mathrm{mmol})$ and $n$-pentadecane as internal GC reference $(0.2 \mathrm{mmol}, 42.5 \mathrm{mg}$ ). The catalyst suspension (see above, $0.4 \mathrm{~mL}$, $0.01 \mathrm{mmol}[\mathrm{Fe}], 5 \mathrm{~mol} \%)$ was added and the vial was closed with a septum. The septum was punctured with a short needle and the vial was transferred to a pressure-resistant reactor equipped with a gas inlet. The reactor was purged with $\mathrm{H}_{2}$ for 1 min while stirring the reaction vial. After three short cycles of (de)pressurizing (2.0 bar, $5 \mathrm{sec}, 1.3 \mathrm{bar}$ ), the $\mathrm{H}_{2}$ pressure and temperature were set $\left(1.3\right.$ bar $\left.\mathrm{H}_{2}, 30^{\circ} \mathrm{C}\right)$. After $5-16 \mathrm{~h}$, the reactor was cooled and the pressure was released. The vials were retrieved and saturated aqueous $\mathrm{NaHCO}_{3}(1.5 \mathrm{~mL})$ and ethyl acetate $(1.5 \mathrm{~mL})$ were added. The resulting suspension was stirred for $20 \mathrm{~min}$, after which the organic phase was separated and filtered through a short $\mathrm{SiO}_{2}$ plug. Yields and selectivities were determined from pure samples obtained by flash column chromatography $\left(\mathrm{SiO}_{2}, n\right.$-pentane, ethyl acetate) or quantitative GC and NMR analyses vs. internal references.

\section{Acknowledgements}

This work was supported by the Priority Program "Materials synthesis at room temperature" of the Deutsche Forschungsgemeinschaft (DFG, SPP 1708). We thank Michaela Lutz and Veronica Scheidler (U Regensburg), Wolfgang Caliebe, Edmund Welter, and the beam line staff of P64 and P65 at PETRA III (DESY, Hamburg) for technical support.

\section{Conflict of interest}

The authors declare no conflict of interest.

Keywords: alkynes · hydrogenation - iron - nanoparticles stereoselectivity

[1] a) A. Fürstner, G. Seidel, J. Organomet. Chem. 2000, 606, 75-78; b) A. Fürstner, F. Stelzer, A. Rumbo, H. Krause, Chem. Eur. J. 2002, 8, 1856 1871 ; c) G. L. Parker, L. K. Smith, I. R. Baxendale, Tetrahedron 2016, 72, 
$1645-1652$; d) E. Breitmeier, Terpene. Aromen, Düfte, Pharmaka, Pheromone, Wiley-VCH, Weinheim, 2005; e) D. Srimani, Y. Diskin-Posner, Y. Ben-David, D. Milstein, Angew. Chem. Int. Ed. 2013, 52, 14131-14134 Angew. Chem. 2013, 125, 14381-14384; f) W.-Y. Siau, Y. Zhang, Y. Zhao Top. Curr. Chem. 2012, 327, 33-58; g) R. H. Grubbs, T. M. Trnka in Ruthenium in Organic Synthesis (Ed.: S.-I. Murahashi), Wiley-VCH, Weinheim, 2004, pp. $153-178$.

[2] a) H. Lindlar, R. Dubuis, Org. Synth. 1966, 46, 89-91; b) H. Lindlar, Helv. Chim. Acta 1952, 35, 446-450.

[3] E. N. Marvell, T. Li, Synthesis 1973, 457-468.

[4] a) F. Alonso, I. Osante, M. Yus, Tetrahedron 2007, 63, 93-102; b) X. Wen, X. Shi, X. Qiao, Z. Wu, G. Bai, Chem. Commun. 2017, 53, 5372-5375 c) J. M. Khurana, A. Gogia, Org. Prep. Proced. Int. 1997, 29, 1-32; d) Z. A. Chobanyan, Russ. J. Appl. Chem. 2004, 77, 2036-2037; e) C. A. Brown, J. Org. Chem. 1970, 35, 1900-1904; f) C. A. Brown, V. K. Ahuja, J. Chem. Soc. Chem. Commun. 1973, 553-554.

[5] C. Chen, Y. Huang, Z. Zhang, X.-Q. Dong, X. Zhang, Chem. Commun 2017, 53, 4612-4615.

[6] a) S. Enthaler, M. Haberberger, E. Irran, Chem. Asian J. 2011, 6, $1613-$ 1623; b) S. C. Bart, E. Lobkovsky, P. J. Chirik, J. Am. Chem. Soc. 2004, 126, 13794-13807; c) C. Belger, B. Plietker, Chem. Commun. 2012, 48, 5419 5421 ; d) M. D. Greenhalgh, A. S. Jones, S.P. Thomas, ChemCatChem 2015, 7, 190-222; e) J. Chen, J. Guo, Z. Lu, Chin. J. Chem. 2018, 36, 1075-1109; M. D. Greenhalgh, D. J. Frank, S.P. Thomas, Adv. Synth. Catal. 2014, 356, 584-590.

[7] a) S.-I. Taira, Bull. Chem. Soc. Jpn. 1962, 35, 840-844; b) K. Hata, I. Mo toyama, K. Sakai, Org. Prep. Proced. Int. 1972, 4, 179-209.

[8] a) T. N. Gieshoff, A. Welther, M. T. Kessler, M. H. G. Prechtl, A. Jacobi von Wangelin, Chem. Commun. 2014, 50, 2261-2264; b) A. Welther, A. Jacobi von Wangelin, Curr. Org. Chem. 2013, 17, 326-335.

[9] a) C. Vollmer, C. Janiak, Coord. Chem. Rev. 2011, 255, 2039-205; b) L. S. Ott, R. G. Finke, Coord. Chem. Rev. 2007, 251, 1075-1100; c) M. Zahmakiran, S. Özkar, Nanoscale 2011, 3, 3462-3481.

[10] T. N. Gieshoff, U. Chakraborty, M. Villa, A. Jacobi von Wangelin, Angew. Chem. Int. Ed. 2017, 56, 3585-3589; Angew. Chem. 2017, 129, 3639 3643.

[11] D. Brenna, M. Villa, T. N. Gieshoff, F. Fischer, M. Hapke, A. Jacobi von Wangelin, Angew. Chem. Int. Ed. 2017, 56, 3585-3589; Angew. Chem. 2017, 129, 3639-3643.

[12] For selected recent reports of stereoselective Z-hydrogenations of alkynes that exhibited good functional group tolerance, see for example: a) A. K. Ghosh, Y. Wang, J. T. Kim, J. Org. Chem. 2001, 66, 8973-8982; b) A. Gollner, K.-H. Altmann, J. Gertsch, J. Mulzer, Chem. Eur. J. 2009, 15, 5979-5997; c) V. Hickmann, M. Alcarazo, A. Fürstner, J. Am. Chem. Soc
2010, 132, $11042-11044$; d) K. Semba, R. Kameyama, Y. Nakao, Synlett 2015, 26, 318-322; e) F. Pape, N. O. Thiel, J. F. Teichert, Chem. Eur. J. 2015, 21, 15934-15938; f) C. F. Heinrich, E. Widemann, J. Sanz, R. Lugan, T. Heitz, F. Pinot, M. Miesch, L. Miesch, Eur. J. Org. Chem. 2015, 1130-1136; g) K. Tokmic, A. R. Fout, J. Am. Chem. Soc. 2016, 138, $13700-13705$.

[13] W. M. Czaplik, S. Grupe, M. Meyer, A. Jacobi von Wangelin, Chem. Commun. 2010, 46, 6350-6352.

[14] A. M. Moiseenkov, B. Schaub, C. Margot, M. Schlosser, Tetrahedron Lett. $1985,26,305-306$.

[15] a) J. A. Widegren, R. G. Finke, J. Mol. Catal. A 2003, 198, 317-341; b) R. H. Crabtree, Chem. Rev. 2012, 112, 1536-1554; c) D. Gärtner, A. L. Stein, S. Grupe, J. Arp, A. Jacobi von Wangelin, Angew. Chem. Int. Ed. 2015, 54, 10545-10549; Angew. Chem. 2015, 127, 10691-10695; d) V. Artero, M. Fontecave, Chem. Soc. Rev. 2013, 42, 2338.

[16] a) A. Welther, M. Bauer, M. Mayer, A. Jacobi von Wangelin, ChemCatChem 2012, 4, 1088-1093; b) M. Bauer, T. Kauf, J. Christoffers, H. Bertagnolli, Phys. Chem. Chem. Phys. 2005, 7, 2664-2670; c) R. B. Bedford, Acc Chem. Res. 2015, 48, 1485-1493; d) C. Cassani, G. Bergonzini, C.-J. Wallentin, ACS Catal. 2016, 6, 1640-1648; e) R. Schoch, W. Desens, T. Werner, M. Bauer, Chem. Eur. J. 2013, 19, 15816-15821; f) H. Takaya, S. Nakajima, N. Nakagawa, K. Isozaki, T. Iwamoto, R. Imayoshi, N. J. Gower, L. Adak, T. Hatakeyama, T. Honma, M. Takagaki, Y. Sunada, H. Nagashima, D. Hashizume, O. Takahashi, M. Nakamura, Bull. Chem. Soc. Jpn. 2015, 88, 410-418; g) A. M. Messinis, S. L. J. Luckham, P. P. Wells, D. Gianolio, E. K. Gibson, H. M. O'Brien, H. A. Sparkes, S. A. Davis, J. Callison, D. Elorriaga, O. Hernandez-Fajardo, R. B. Bedford, Nat. Catal. 2019, 2, $123-$ 133.

[17] For details, see the Supporting Information.

[18] a) R. W. G. Wyckoff, Cryst. Struct. 1963, 1, 7; b) Lattice parameter of FeAl-alloys: 0.286-0.293 nm, A. N. Sinha, Trans. J. I. M. 1984, 25, 900-901 c) Interplanar spacing of $\mathrm{Fe}_{3} \mathrm{O}_{4}: 0.251 \mathrm{~nm}, \mathrm{H}$. E. Ghandoor, H. M. Zidan, M. M. H. Khalil, M. I. M. Ismail, Int. J. Electrochem. Sci. 2012, 7, 5734 5745 ; d) Interplanar spacing of $\alpha-\mathrm{Fe}_{2} \mathrm{O}_{3}: 0.171 \mathrm{~nm}$ (116 plane), Interplanar spacing of $\gamma-\mathrm{Fe}_{2} \mathrm{O}_{3}: 0.211 \mathrm{~nm}$ (311 plane), $0.253 \mathrm{~nm}$ (220 plane), R. A. Bepari, P. Bharali, B. K. Das, J. Saudi Chem. Soc. 2017, 21, S170S178.

Manuscript received: April 4, 2019

Revised manuscript received: June 4, 2019

Accepted manuscript online: July 2, 2019

Version of record online: July 25, 2019 